\title{
Expresión de óxido nítrico sintetasa en mucosa sinusal de pacientes portadores de sinusitis crónica versus controles
}

\section{Expression of nitric oxide synthase in sinus mucosa of chronic sinusitis patients vs controls}

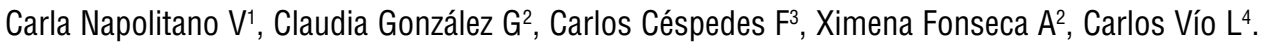

\begin{abstract}
RESUMEN
Introducción: El óxido nítrico producido en las cavidades paranasales juega un importante rol en la fisiología nasal ya que aumenta la frecuencia del batido ciliar, optimiza el barrido mucociliar y tiene un efecto bacteriostático y virustático.

Diversos estudios muestran que el óxido nítrico nasal exhalado en pacientes con rinosinusitis crónica es significativamente más bajo que en sujetos sanos. Esto podría estar determinado por una alteración en la difusión del óxido nítrico a través de los ostia de drenaje obstruidos o por una menor producción de óxido nítrico por parte de los portadores de rinosinusitis crónica. En este último caso, esto podría corresponder al evento primario en la etiopatogenia de la rinosinusitis crónica.

Objetivos: Estudiar y comparar cualitativamente con técnica de inmunohistoquímica la expresión de las isoformas e-NOS e i-NOS de la óxido nítrico sintetasa en mucosa sinusal de pacientes con rinosinusitis crónica y pacientes controles, sin patología rinosinusal infecciosa o alérgica.

Material y método: Para responder a esta interrogante se realizó un estudio comparativo de casos y controles, con el objetivo de cuantificar con técnica de inmunohistoquímica la expresión de las isoformas endotelial e inducible de la óxido nítrico sintetasa en mucosa sinusal de pacientes con rinosinusitis crónica y pacientes controles sin patología rinosinusal infecciosa o alérgica.

Resultados: Ingresan al estudio un total de 11 pacientes. Seis de ellos son el grupo control y 5 con sinusitis crónica. Ambos grupos presentan resultados similares.

Conclusión: Los resultados no muestran ninguna diferencia en la expresión de óxido nítrico sintetasa, tanto en su isoforma endotelial como inducible, en la mucosa sinusal de pacientes portadores de rinosinusitis crónica comparado con sujetos sanos.

Palabras clave: óxido nítrico, expresión óxido nítrico sintetasa.
\end{abstract}

\begin{abstract}
Introduction. Nitric oxide produced in the paranasal sinuses plays an important role in nasal physiology because it increases ciliary beat frequency, improves mucociliary clearance and has a bacteriostatic and virustatic effect.

\footnotetext{
Médico Jefe Servicio de Otorrinolaringología Hospital Regional de Talca.

Médico, Departamento de Otorrinolaringología, Hospital Clínico de la Pontificia Universidad Católica de Chile.

Tecnólogo médico Facultad Ciencias Biológicas, Universidad Católica de Chile.

Profesor Titular Facultad Ciencias Biológicas, Universidad Católica de Chile.
} 
Several studies have shown that exhaled nasal nitric oxide was substantially lower in chronic sinusitis patients, compared to healthy subjects. This could be determined by altered nitric oxide diffusion through obstructed drainage ostia, or by chronic sinusitis patients having lower nitric oxide production.

Aims. To qualitatively compare by immunocytochemistry the expression of the iNOS and eNOS isoforms of nitric oxide synthase in the sinus mucosa of chronic sinusitis patients and control subjects.

Materials and methods. A case-control comparative study was carried out in order to compare by immunocytochemistry the expression of inducible and epithelial isoforms of nitric oxide synthase in nasal mucosa of chronic sinusitis patients and control subjects, with no infectious or allergic rhinosinusal pathology.

Results. The results show no difference in the expression of the inducible or epithelial isoform of nitric oxide synthase in the nasal mucosa of chronic sinusitis patients as compared to healthy subjects.

Key word. Nitric oxide, nitric oxide synthase expression.Introducción

\section{INTRODUCCIÓN}

El óxido nítrico (ON) está involucrado en diversos procesos fisiológicos del organismo, como la regulación del tono vascular, la actividad plaquetaria y la neurotransmisión, tanto a nivel del sistema nervioso central como periférico, entre otros ${ }^{1}$. Particularmente en la vía aérea, el ON ha demostrado tener un importante efecto bacteriostático ${ }^{2}$ y virustátic $0^{3}$, estimular el barrido mucociliar aumentando la frecuencia del batido ciliar ${ }^{4,5}$, ser mediador de la inflamación $n^{6}$ y regular el tono bronquial y vascular pulmonar ${ }^{7,8}$. En condiciones normales en la vía aérea superior, el $\mathrm{ON}$ es producido principalmente por las células epiteliales ciliadas de las cavidades paranasales, y en menor cantidad por la mucosa nasal ${ }^{8}$.

EI ON se genera a partir de la transformación del aminoácido L-arginina en L-citrulina, proceso realizado por la enzima óxido nítrico sintetasa (NOS). Esta enzima presenta tres isoformas conocidas: Tipo I 0 neuronal (nNOS) y Tipo III o endotelial (eNOS), que se expresan en forma constitutiva en las células epiteliales de la vía aérea; y la isoforma inducible 0 Tipo II (iNOS) ${ }^{9}$. Existen varias diferencias entre ellas. Las eNOS y nNOS son dependientes del sistema calcio-calmodulina para su activación, no son inhibidas por corticosteroides y producen pequeñas cantidades de $0 \mathrm{~N}^{10}$, mientras que la iNOS es independiente del sistema calcio-calmodulina, es activada por citoquinas proinflamatorias y endotoxinas, es inhibida por corticosteroides y produce hasta 1000 veces más ON que las isoformas constitutivas ${ }^{10}$.
Mediante estudios con técnica de inmunohistoquímica ${ }^{11}$ se ha podido determinar que las tres isoformas de óxido nítrico sintetasa (endotelial, inducible y neuronal) se expresan constitutivamente en el epitelio sinusal humano sin patología. Estudios posteriores han demostrado que la iNOS presente en las cavidades paranasales posee otras diferencias con las iNOS de otra ubicación, además de expresarse constitutivamente: es dependiente del sistema calciocalmodulina y no es inhibida por corticosteroides ${ }^{10}$. Eso sí, la iNOS nasal produce grandes cantidades de ON, al igual que otras iNOS.

La producción de ON puede ser modificada por factores ambientales, ya que se ha observado una disminución del ON exhalado en pacientes tabáquicos ${ }^{12}$, y con el uso de descongestionantes tópicos como oximetazolina $^{13}$. No se han reportado alteraciones en la producción de ON con el uso de esteroides tópicos 0 sistémicos 0 antimicrobianos en pacientes sanos ${ }^{14}$.

La etiopatogenia de la rinosinusitis crónica aún no se conoce a cabalidad. Determinaciones del óxido nítrico nasal exhalado, medido por quimioluminiscencia, han demostrado una disminución de la medición de ON exhalado nasal en pacientes con rinosinusitis crónica comparado con sujetos sanos y pacientes con resfrío común ${ }^{15,16}$, lo que se correlacionó con una disminución de la frecuencia de batido ciliar ${ }^{17}$. Se desconoce la causa de esta alteración, pero se postula que podría estar determinada por una alteración en la difusión del ON a través de los ostia de drenaje obstruidos, si bien no se puede descartar que se deba a una menor producción de ON por parte de la mucosa sinusal de estos pacientes, ya que no existen 
estudios inmunohistoquímicos que cuantifiquen la expresión de la enzima óxido nítrico sintetasa en pacientes con rinosinusitis crónica versus pacientes sanos.

Para responder esta interrogante se diseñó un estudio con el objetivo de estudiar y comparar cualitativamente con técnica de inmunohistoquímica la expresión de las isoformas e-NOS e i-NOS de la óxido nítrico sintetasa en mucosa sinusal de pacientes con rinosinusitis crónica y pacientes controles, sin patología rinosinusal infecciosa 0 alérgica.

Si nuestros resultados mostraran que la disminución del ON nasal exhalado en pacientes con rinosinusitis crónica comparado con sujetos sanos se debe a una menor producción de ON por parte de la mucosa sinusal, cabe preguntarse ¿es éste el evento primario en la etiopatogenia de la rinosinusitis crónica?

\section{MATERIAL Y MÉTODO}

Se realizó un estudio comparativo de casos y controles, con análisis ciego, en conjunto entre el Hospital Clínico de la Pontificia Universidad Católica de Chile y el Laboratorio de Ciencias Fisiológicas de la Facultad de Ciencias Biológicas de la Pontificia Universidad Católica de Chile, entre diciembre de 2003 y octubre 2004. Todos los pacientes incluidos en el estudio leyeron y firmaron un consentimiento informado que autoriza al equipo médico a examinar, disponer y utilizar los tejidos u órganos extirpados, con propósitos científicos 0 docentes.

El grupo en estudio está constituido por pacientes mayores de 18 años con diagnóstico de rinosinusitis crónica (clínico y radiológico con Tomografía Computada de Cavidades Paranasales), con indicación quirúrgica definida por ausencia de respuesta a tratamiento médico (2 cursos de 30 días de antibióticos de segunda línea, corticoides tópicos y orales, y lavados nasales), corroborada por Tomografía de Cavidades Paranasales, que muestre compromiso sinusal después de completado el tratamiento. Durante la cirugía endoscópica de cavidades paranasales con la técnica de Messerklinger se tomó una biopsia de mucosa del seno paranasal más afectado.

El grupo control está formado por pacientes mayores de 18 años, con diagnóstico de adenoma hipofisiario con indicación de abordaje transesfe- noidal de hipófisis, y por pacientes con indicación de descompresión orbitaria endoscópica, en ambos casos con Tomografía Computada de Cavidades Paranasales prequirúrgica normal. Durante la cirugía (abordaje transesfenoidal de hipófisis o descompresión orbitaria endoscópica) se toma muestra de mucosa sana de seno paranasal.

Se excluyeron del estudio los pacientes con antecedentes de tabaquismo, cirugía nasal 0 de cavidades paranasales previa, clínica de rinitis alérgica y/o test cutáneo positivo, uso de oximetazolina en aerosol nasal en el último mes, y poliposis nasal al examen físico o Tomografía Computada de Cavidades Paranasales.

Una vez obtenidas las muestras, éstas fueron inmediatamente fijadas en paraformaldehído al $4 \%$, incluidas en parafina y cortadas con un grosor de $6 \mu \mathrm{m}$. Las secciones fueron incubadas en el anticuerpo primario (anti-eNOS y anti-iNOS monoclonal de ratón, Transduction Laboratories) a una dilución de 1:50 por 2 horas y enjuagadas en PBS. Luego fueron incubadas con el anticuerpo secundario (inmunoglobulina cabra antirratón, Transduction Laboratories) por 30 minutos y enjuagadas en PBS. Para la tinción, las secciones se hicieron reaccionar con peroxidasa de estreptavidina por 30 minutos y se enjuagaron en PBS. Finalmente fueron tratadas con solución sustrato-cromogena por 3 minutos y montadas después de tinción control con hematoxilina.

\section{RESULTADOS}

Ingresaron al estudio un total de 11 pacientes: 6 pacientes al grupo control, y 5 al grupo de sinusitis crónica. De los 6 pacientes controles, 5 proceden de abordajes transesfenoidales de hipófisis y 1 de una descompresión orbitaria por orbitopatía de Graves. Las edades promedio fueron de 37 años en el grupo control y 45 años en el grupo sinusitis crónica. En el grupo control el $33,3 \%$ de la muestra fue de género femenino, mientras que en el grupo sinusitis crónica, el $60 \%$ de la muestra fue de género femenino.

El análisis bajo microscopía de luz de los cortes de mucosa sinusal de los pacientes controles reveló una intensa inmunoreactividad de las isoformas endotelial (eNOS) (Figura 1) e inducible 


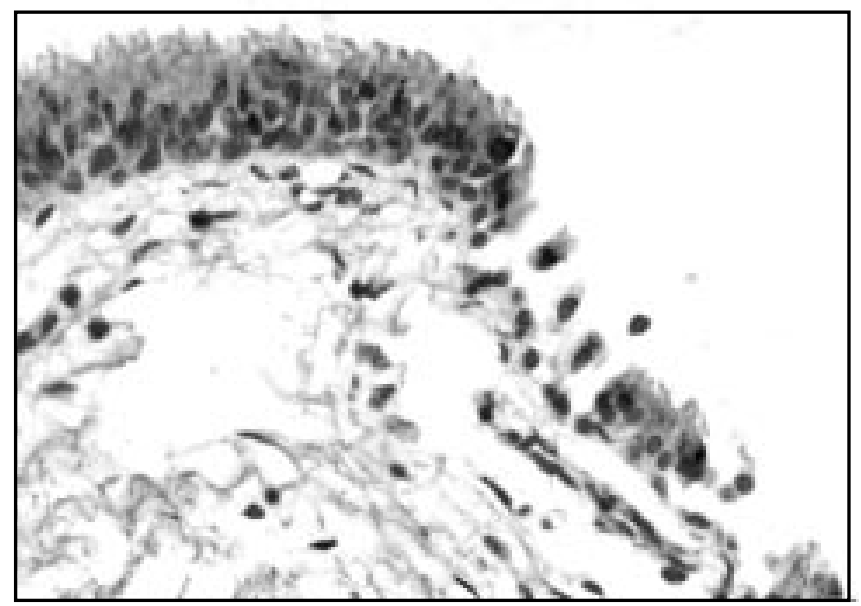

Figura 1: Paciente control, tinción (+) para eNOS, patrón citoplasmático.

(iNOS) de óxido nítrico sintetasa. Esta tinción se observó en las células epiteliales ciliadas, glándulas de la submucosa y células endoteliales de los vasos de la submucosa, indistintamente para ambas isoformas de la enzima. Las células epiteliales ciliadas presentaron dos patrones distintos de tinción inmunohistoquímica, lo que se observó para ambas isoformas: 1) un patrón citoplasmático difuso (Figura 1), y 2) un patrón ciliar (Figura 2), en el cual la tinción compromete exclusivamente el borde apical ciliado del epitelio.

La microscopía de luz de la mucosa sinusal del grupo portador de sinusitis crónica reveló en 2 casos $(40 \%)$ una infiltración inflamatoria crónica de la submucosa, asociada a epitelio sinusal inmaduro de tipo regenerativo (disminución o ausencia de células columnares ciliadas).

Al igual que en los sujetos controles, en la mucosa sinusal de la totalidad de los pacientes portadores de sinusitis crónica se detectó una intensa inmunorreactividad de óxido nítrico sintetasa, tanto de eNOS como de iNOS (Figura 3), en las células epiteliales ciliadas, glándulas de la submucosa y células endoteliales de los vasos de la submucosa. Las células epiteliales ciliadas de los pacientes con sinusitis crónica también presen-

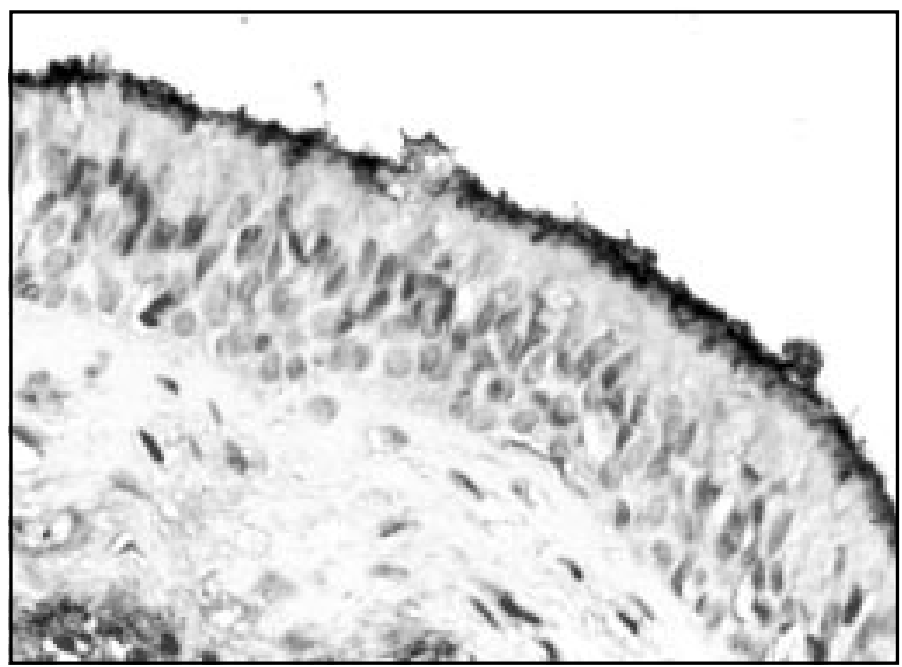

Figura 2: Paciente control, tinción (+) para iNOS, patrón ciliar. 


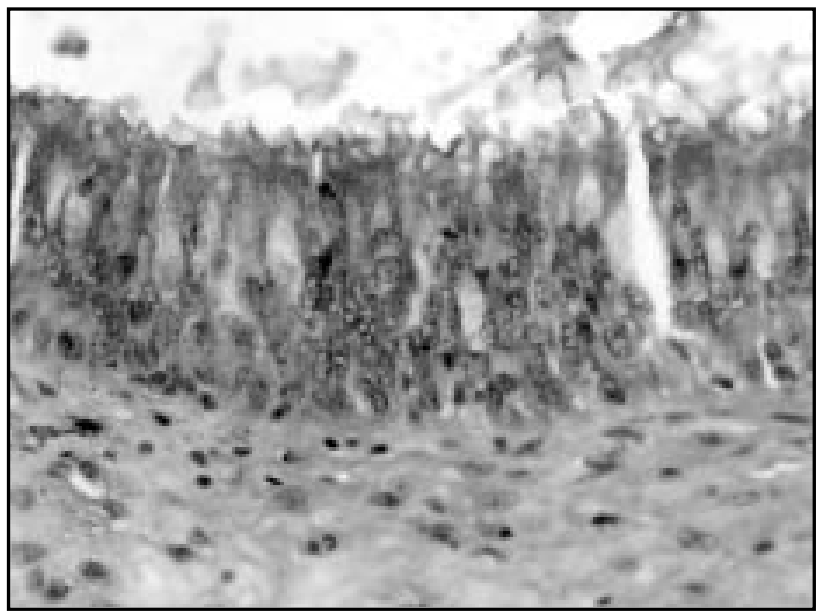

Figura 3: Paciente portador de sinusitis crónica, tinción (+) para iNOS, patrón citoplasmático y ciliar.

taron los dos patrones de tinción inmunohistoquímica descritos en los pacientes controles para ambas isoformas: el patrón citoplasmático y el patrón ciliar, excepto en los dos pacientes que mostraron un epitelio sinusal inmaduro con disminución 0 ausencia de células columnares ciliadas, en cuyo caso no se observó el patrón de tinción ciliar, ya que se tiñó el citoplasma de células epiteliales de aspecto inmaduro.

\section{DISCUSIÓN}

No se encontraron diferencias cualitativas al comparar la expresión de óxido nítrico sintetasa (NOS) en la mucosa sinusal de pacientes sanos versus pacientes portadores de sinusitis crónica, tanto en relación al tipo de célula que expresa la enzima (células epiteliales ciliadas, glándulas de la submucosa y células endoteliales de los vasos de la submucosa), a la isoforma de NOS expresada (iNOS y eNOS) y al patrón de expresión de NOS (citoplasmático y ciliar), exceptuando en este último caso, a los 2 pacientes que presentaron inmadurez de las células columnares ciliadas, y por lo tanto, ausencia de patrón ciliar.

Considerando estos resultados, nuestro estudio no apoya la hipótesis de una menor producción de ON por parte de la mucosa de los pacientes portadores de sinusitis crónica, sino que más bien de una menor llegada de ON al sitio de medición de éste, la cavidad nasal, lo que podría explicarse por una menor difusión del ON a la cavidad nasal secundaria a edema de la mucosa sinusal, obstrucción de los ostia de drenaje de los senos paranasales propio del proceso de rinosinusitis, 0 efecto de presión negativa al interior del seno mal ventilado.

La isoforma inducible de la óxido nítrico sintetasa (iNOS) es más eficiente en la producción de $\mathrm{ON}$ que las isoformas endotelial y neuronal. De esta manera, un cambio en la proporción de la expresión de las distintas isoformas, predominando las menos eficientes, podría haber explicado las bajas mediciones de $\mathrm{ON}$ exhalado nasal en sinusitis crónica, pero tampoco encontramos ninguna diferencia en el tipo de isoforma expresada entre ambos grupos.

La demostración de la presencia de óxido nítrico sintetasa en la mucosa sinusal de los pacientes portadores de sinusitis crónica abre nuevas perspectivas terapéuticas, haciendo posible el tratamiento de esta enfermedad con dadores de óxido nítrico, como la L-arginina, ya que el requisito para la conversión de L-arginina en ON es la presencia de la enzima que cataliza esta reacción. De esta forma se podría potenciar el batido ciliar y el efecto bacteriostático y virustático que el ON tiene sobre el epitelio sinusal en los pacientes portadores de rinosinusitis crónica.

El número total de pacientes que ingresó al estudio fue bajo, dado por la relativa baja inciden- 
cia de las patologías estudiadas (abordaje transesfenoidal de hipófisis, descompresión orbitaria, sinusitis crónica con indicación quirúrgica), y a los variados y prevalentes criterios de exclusión (rinitis alérgica, tabaquismo, etc...). A pesar de ello, fue suficiente para nuestro análisis cualitativo, ya que todos los pacientes del grupo sinusitis crónica presentaron inmunoreactividad para ambas isoformas de NOS.

\section{CONCLUSIÓN}

Este estudio demuestra la presencia constitutiva de la enzima óxido nítrico sintetasa, en sus isoformas endotelial e inducible (eNOS e iNOS), en la mucosa sinusal humana normal.

La mucosa sinusal de los pacientes portadores de rinosinusitis crónica expresa ambas isoformas de óxido nítrico sintetasa, eNOS e iNOS, sin diferencias cualitativas con respecto a los pacientes controles. Este hallazgo hace factible el uso terapéutico de dadores de óxido nítrico, como la Larginina, en rinosinusitis crónica.

De acuerdo a esto, no habría diferencias en la producción de óxido nítrico entre sanos y portadores de rinosinusitis crónica. La disminución observada en la medición de ON exhalado nasal en pacientes con rinosinusitis crónica comparado con sujetos sanos se debería a una menor difusión del ON desde las cavidades paranasales a la cavidad nasal.

\section{Agradecimientos}

A la Sociedad Chilena de Otorrinolaringología, Medicina y Cirugía de Cabeza y Cuello y a su Comité de Investigación y Desarrollo, por el financiamiento otorgado para la realización de este proyecto.

Al Dr. Jorge Caro Letelier por su aporte en la inclusión de pacientes controles a este estudio.

\section{BIBLIOGRAFÍA}

1. Singh S, Evans TW. Nitric oxide, the biological mediator of the decade: fact or fiction? Eur Resp J 1997; 10: 699-707.
2. Nathan CF, HibBs JB. Role of nitric oxide synthesis in macrophage antimicrobial activity. Curr Opin Immunol 1991; 3: 65-70.

3. CROEN KD. Evidence for an antiviral effect of nitric oxide. J Clin Invest 1993; 91: 2446-52.

4. Runer T, Cervin A, Lindberg $S$ et al. Nitric oxide is a regulator of mucociliary activity in the upper respiratory tract. Otolaryngol Head Neck Surg 1998; 119: 278-87.

5. Yang B, Schlosser RJ, McCaffrey TV. Dual signal transduction mechanisms modulate ciliary beat frequency in upper airway epithelium. Am J Physiol 1996; 270: L745-L751.

6. Lundberg JON, Weitzberg E, Lundberg JM, Alving K. Nitric oxide in exhaled air. Eur Respir J 1996; 9: 2671-80.

7. Al-Alı MK, Howarth PH. Nitric oxide and the respiratory system in health and disease. Resp Med 1998; 92: 701-15.

8. Lundberg JON, Weitzberg E, Nordvall SL, Kuylenstierna R, Lundberg JM, Alving K. Primarily nasal origin of exhaled nitric oxide and absence in Kartagener's syndrome. Eur Respir J 1994; 7: 1501-4.

9. Lefevere L, Willems T, Lindberg $S$, Jorissen M. Nasal nitric oxide. Acta oto-rhino-laryngologica belg 2000; 54: 271-80.

10. DJupesland PG, Chatkin JM, QIAN W, Haight JSJ. Nitric Oxide in the nasal airway: a new dimension in otorhinolaryngology. $\mathrm{Am} \mathrm{J}$ Otolaryngol 2001; 22: 19-32.

11. Jeong-Whun K, Yang-Gi M, Chae-Seo R et al. Regulation of mucociliary motility by nitric oxide and expression of nitric oxide synthase in the human sinus epithelial cells. Laryngoscope 2001; 111: 246-50.

12. Olin AC, Hellgren J, Karlsson G, Luungkvist G, Nolkrantz K, Toren K. Nasal nitric oxide and its relationship to nasal symptoms, smoking and nasal nitrate. Rhinology 1998; 36: 117-21.

13. Westerveld GJ, Voss hP, Van der Hee RM. Inhibition of nitric oxide synthase by nasal decongestants. Eur Respir J 2000 Sep; 16(3): 437-44.

14. Lundberg J0, Weitzberg E, Rinder J et al. Calciumindependent and steroid-resistant nitric oxide synthase activity in human paranasal sinus mucosa. Eur Respir J 1996; 9: 1344-7. 
15. Arnal JF, Flores P, Rami J et al. Nasal nitric oxide concentration in paranasal sinus inflammatory diseases. Eur Respir J 1999; 13: 307-12.

16. Lindberg S, Cervin A, Runer T. Nitric oxide (NO) production in the upper airways is decreased in chronic sinusitis. Acta Otolaryngol (Stockh) 1997 Jan; 117: 113-7.

17. Lindberg S, Cervin A, Runer T. Low levels of nasal nitric oxide (NO) correlate to impaired mucociliary function in the upper airways. Acta Otolaryngol (Stockh) 1997; 117: 728-34. 